\title{
A highly conserved molecular switch binds MSY-3 to regulate myogenin repression in postnatal muscle
}

\author{
Libera Berghella, ${ }^{1,2,5,9}$ Luciana De Angelis, ${ }^{3}$ Tristan De Buysscher, ${ }^{2}$ Ali Mortazavi, ${ }^{2}$ \\ Stefano Biressi, ${ }^{4,7}$ Sonia V. Forcales, ${ }^{1,6}$ Dario Sirabella, ${ }^{1,3,8}$ Giulio Cossu, ${ }^{1,4}$ and Barbara J. Wold ${ }^{2}$ \\ ${ }^{1}$ Institute of Cell Biology and Tissue Engineering, San Raffaele Biomedical Science Park, Rome 00128, Italy; ${ }^{2}$ Division of \\ Biology, California Institute of Technology, Pasadena, California 91125, USA; ${ }^{3}$ Department of Histology and Medical \\ Embryology, University "La Sapienza," Rome 00161, Italy; ${ }^{4}$ Stem Cell Research Institute, Dibit, H. San Raffaele, \\ Milan 20132, Italy
}

\begin{abstract}
Myogenin is the dominant transcriptional regulator of embryonic and fetal muscle differentiation and during maturation is profoundly down-regulated. We show that a highly conserved 17-bp DNA cis-acting sequence element located upstream of the myogenin promoter (myogHCE) is essential for postnatal repression of myogenin in transgenic animals. We present multiple lines of evidence supporting the idea that repression is mediated by the Y-box protein MSY-3. Electroporation in vivo shows that myogHCE and MSY-3 are required for postnatal repression. We further show that, in the $\mathrm{C} 2 \mathrm{C} 12$ cell culture system, ectopic MSY-3 can repress differentiation, while reduced MSY-3 promotes premature differentiation. MSY-3 binds myogHCE simultaneously with the homeodomain protein $\mathrm{Pbx}$ in postnatal innervated muscle. We therefore propose a model in which the myogHCE motif operates as a switch by specifying opposing functions; one that was shown previously is regulated by $\mathrm{MyoD}$ and $\mathrm{Pbx}$ and it specifies a chromatin opening, gene-activating function at the time myoblasts begin to differentiate; the other includes MYS-3 and Pbx, and it specifies a repression function that operates during and after postnatal muscle maturation in vivo and in myoblasts before they begin to differentiate.
\end{abstract}

[Keywords: CsdA; MSY-3; myogenin; Y-box; innervation; muscle maturation]

Supplemental material is available at http://www.genesdev.org.

Received January 3, 2008; revised version accepted June 4, 2008.

Muscle-specific transcription factors of the MyoD family (MRFs) are members of a regulatory network that drives skeletal muscle determination and differentiation during embryonic and fetal development. Among the four MRFs, myogenin is critical because it is the one required for correct differentiation of virtually all embryonic and fetal muscle. Germline ablation of myogenin blocks differentiation and null mice die at birth from lack of muscle fiber development and consequent muscle insufficiency (Hasty et al. 1993; Nabeshima et al. 1993). Myogenin is expressed prominently at the onset of myocyte differentiation as embryonic and fetal development proceed, with recruitment of new myoblasts to the differ-

Present addresses: ${ }^{5}$ Institute of Cell Biology and Tissue Engineering, San Raffaele Biomedical Science Park, Rome 00128, Italy; ${ }^{6}$ The Burnham Institute, La Jolla, CA 92037, USA, ${ }^{7}$ Department of Neurology and Neurological Sciences, Stanford University School of Medicine, Stanford, CA 94305, USA; ${ }^{8}$ Department of Developmental and Regenerative Biology, Mount Sinai School of Medicine, New York, NY 10029 USA.

${ }^{9}$ Corresponding author.

E-MAIL libera.berghella@spr-r.it; FAX 39-06-80319054.

Article is online at http://www.genesdev.org/cgi/doi/10.1101/gad.468508. entiated state continuing throughout this period and into the early postnatal period (Sassoon et al. 1989; Wright et al. 1989; Hannon et al. 1992). In healthy wildtype adult muscle, myogenin is down-regulated, as muscle matures, around the time of innervation, but it can be up-regulated later during muscle regeneration in response to diverse stimuli (e.g., Grounds et al. 1992), during aging (Musarò et al. 1995; Kostrominova et al. 2000), and upon denervation (Eftimie et al. 1991; Buonanno et al. 1992). Knocking out myogenin perinatally leads to a generally normal skeletal muscle phenotype, but with reduced body size (Knapp et al. 2006).

At the molecular level, myogenin interacts with many muscle-specific target genes, associated with muscle differentiation, including $n A C h R$ subunits, by binding to one or more E-boxes (5'-CANNTG-3') present in their promoters and transcriptional enhancers (Piette et al. 1990; Prody and Merlie 1991; Berberich et al. 1993; Walke et al. 1996). During postnatal development the major functional maturation event is motor innervation, which activates a cascade of calcium-dependent signals, leading to overall reduced myogenin and MyoD RNA 
levels. This is thought to be important for reducing expression in extrasynaptic regions of synaptic proteins such as AChR subunits, which are positively regulated throughout the fiber by $\mathrm{MyoD}$ and myogenin during early muscle development but later become restricted to synaptic regions during and after innervation (Sanes and Lichtman 2001). When challenged by denervation, myogenin is up-regulated, and its target AChR RNAs and proteins reappear in extrasynaptic regions along the muscle fiber.

How neural activity controls myogenin in postnatal muscle has been the subject of several studies that suggest contributions from multiple mechanisms. A posttranslational pathway has been reported in which extracellular calcium enters through voltage-activated channels and increases activities of PKC and CaMKII, leading to phosphorylation of myogenin protein. This post-translational modification inhibits myogenin binding to Eboxes on $n A C h R$ promoters, resulting in reduced $n A C h R$ expression (Mendelzon et al. 1994; Macpherson et al. 2002). Subsequently, it was proposed that an additional but otherwise unspecified transcriptional mechanism also inhibits myogenin expression in response to innervation (Blagden et al. 2004). The post-translational mechanism could act on a rapid time scale and, among other activities, it could disrupt the positive autoregulatory feedback of myogenin on its own enhancer. Recent studies have further probed transcriptional repression of myogenic target genes that involves recruiting HDAC complexes. So far, two well-known sequence-specific DNA-binding transcription factors, MEF2 and Six-1, have been shown to interact with different histone deacetylases HDACs (Mejat et al. 2005; Tang and Goldman 2006; Cohen et al. 2007), and there are conserved sites for both of these regulators in the 1-kb proximal enhancer/promoter region of myogenin that has been studied in some detail (Edmondson et al. 1992; Cheng et al. 1993; Yee and Rigby 1993; Spitz et al. 1998). The Mef2 and Six transcription factor/corepressor/HDAC sets appear to contribute some partial repression of myogenin, and each can apparently be converted to a positive regulatory complex by replacing corepressors/HDAC activity with coactivators.

Additional regulatory sequences in the myogenin gene have been identified between -1102 and -1565 , and these respond to experimental denervation in the adult (Buonanno et al. 1993; Gibney and Buonanno 1995).

In this work, we show that a third DNA motif, which is located within the 1.1-kb myogenin proximal regulatory region and positioned upstream of the MRFs, MEF2, and Six sites, mediates profound postnatal down-regulation of myogenin RNA. We were initially motivated to study this element by results of comparative genome sequence analysis, which showed it to be highly conserved (myogHCE). This sequence region was independently shown to bind the homeodomain protein $\mathrm{Pbx}$, in close collaboration with MyoD. It contributes to positive regulation of myogenin at the onset of its expression in differentiating (Berkes et al. 2004). We now find that this motif has an alternative and strong repressing regulatory action in postnatal muscle maturation that is associated with binding of MSY-3 in vitro and in vivo.

MSY-3 was not previously known to be part of the core muscle gene regulatory network. It is a member of the cold-shock domain (CSD) family of proteins (also known as Y-box proteins). CSD proteins are characterized by a common DNA/RNA-binding region called the "coldshock domain," which is conserved from bacteria to mammals (Graumann and Marahiel 1998; Matsumoto and Wolffe 1998). Experiments reported here suggest that MSY-3 mediates the normal postnatal down-regulation of myogenin that is associated with innervation. We also find that, in the $\mathrm{C} 2 \mathrm{C} 12$ cell culture system, elevated MSY-3 levels can repress expression of differentiation of specific genes, while reduced MSY-3 can promote premature differentiation. We therefore consider implications of a compound DNA motif capable of binding two different complexes-one including MyoD and $\mathrm{Pbx}$ and executing a chromatin opening, gene-activating function, and another at a later time in normal development, that includes MSY-3 and executes a repression function.

We discuss the implications for sequence conservation of any situation in which multiple factor recognition supports different functions, such as the switch from an early myogenin-on state in developing myocytes to a later myogenin-off state in mature innervated muscle fibers.

\section{Results}

Multispecies comparative genome analysis highlights a myogenin promoter conserved element

An especially conserved sequence feature (myogHCE) was highlighted using MUSSA, a software tool that automatically integrates multigenome genome comparisons and allows for interactive visualization (http://mussa. caltech.edu). This highly conserved element is defined by a core motif of $17 \mathrm{bp}$ shared perfectly across five mammalian genomes (Supplemental Fig. 1A) and surprisingly was also found identical to mammals in pufferfish genomes (Supplemental Fig. 1B). The sequence is located between -101 and $-117 \mathrm{bp}$ upstream of the myogenin transcription start in mouse (chr1: 136,106,084$136,106,132)$ and falls within the 1.0 -kb proximal promoter/enhancer that is sufficient to recapitulate the major features of myogenin expression during embryonic and fetal development (Yee and Rigby 1993).

We next asked if there are other sequences in the genome that are very similar to the myogHCE and also conserved in multiple mammalian genomes. To do this we first retrieved the corresponding sequence from additional vertebrates (Supplemental Fig. 1B). This introduced evolutionary variation, which enabled us to build a position-specified frequency matrix (PSFM) model for myogHCE (Supplemental Material; Mortazavi et al. 2006). The purpose of a PSFM model is to capture allowed variation in a motif. This makes possible computational searches for similar sequences that are scored 
and ranked in a way that is sensitive to the probability of a given nucleotide at each position.

The resulting PSFM model for the myogenin HCE was used to search genome-wide for additional high scoring sites that passed a threshold ( 0.9 match score) in each of three mammalian genomes (human, mouse, and dog), as described previously. We then identified the gene associated with each of these instances by applying the cisMatcher algorithm (Mortazavi et al. 2006). There were only 19 such highly conserved instances associated with orthologous genes in the three genomes (Supplemental Table 1). One of these was located $\sim 35 \mathrm{~kb}$ upstream of mouse $M y o D$ (-32 kb in human) where it is embedded in a larger conserved, noncoding region that has not, to our knowledge, been studied previously. The PSFM model was converted to a conventional consensus sequence to permit direct scanning of Transfac. No perfect matches were found to known consensus sites in that database.

\section{MyogHCE affects myogenin and AChRs extrasynaptic expression during postnatal muscle maturation}

We first tested for functional activity of the myogHCE by constructing a set of lentiviral LacZ reporter genes containing wild-type or mutant myogHCEs, in the context of the $1.1-\mathrm{kb}$ myogenin promoter that drives expression in embryo and fetal muscle (Cheng et al. 1993; Yee and Rigby 1993; Venuti et al. 1995). In each of two independent mutant reporter constructs, the myogHCE was replaced by a different randomized sequence of base composition similar to myogHCE (Supplemental Fig. 2). Prior work had shown that a wild-type 1.1-kb myogenin promoter, in a lentiviral vector, drives GFP expression in a pattern that nicely recapitulates RNA in situ data at day 11.5 of embryonic development (Lois et al. 2002). Initial experiments compared each mutated version, MYOmutL and MYOmutT, with the wild-type (MYOwt) version in embryos. $\beta$-gal staining of 10.5-, 11.5-, and 12.5-d-post-coitum embryos delivered strong myogenin patterns from the wild-type construct in spatiotemporal domains in which populations of myoblasts are continually and actively turning on myogenin in this period, including newer somites of the axis and the limbuds. The mutant construct's staining patterns were not detectably different from the wild-type construct (Supplemental Fig. 3; see the Discussion).

We then asked if mutation of the HCE affects expression in postnatal and adult muscle. Wild-type reporter gene expression was prominent in the first weeks of postnatal life, in agreement with previous studies using conventional transgenesis, and it then declines (Buonanno et al. 1993). Muscle cross-sections from 1- to 5-mo-old animals show progressively less LacZ staining. This tracks the temporal pattern of endogenous myogenin and AChR expression in postnatal life. Furthermore, after $>1 \mathrm{yr}$, some myonuclei again score positive, but at low frequency. This agrees with previously reported "re-expression" of myogenin in aged mice (Musarò et al. 1995). In contrast, cross-sections and muscle fibers from the mutant HCE reporter transgenics,
MYOmutL, showed strong persistence of expression through the period of postnatal maturation (Fig. 1A; Supplemental Fig. 4A). RNA levels were directly quantified by qRT-PCR in MYOmutL and MYOmutT, confirming that the postnatal wild-type reporter reduction occurs at the RNA level, that it mirrors diminution in endogenous myogenin RNA (Fig. 1B; Supplemental Fig. $4 \mathrm{~B})$, and that an intact myogHCE is required for this down-regulation (Supplemental Table 2). We used transgenic mouse lines derived by conventional methods to confirm that expression of the HCE mutant reporter persists in postnatal life. Moreover, no differences in mutant reporter expression were seen upon experimental denervation (Fig. 1C; Supplemental Fig. 4C).

We next established that, in a standard acute transfection assay in healthy adult muscle, a LacZ reporter driven by HCE mutant myogenin promoters shows the expected increase of LacZ positive myonuclei compared with the corresponding wild-type HCE-driven construct (Fig. 1D). Results were identical for the two different mutant constructs. We could then ask whether expression of the myogenin-coding sequence, identically driven by the mutant HCE-containing myogenin promoter, could alter expression of known innervation-dependent endogenous genes, as one would predict if HCEmediated regulation is critical for innervation-dependent regulation. Myogenin and $\alpha$ AChR RNA and protein expression increased in muscle containing the HCE mutant-driven myogenin, but not with the control wildtype myogenin construct (Fig. 2A). Since it is known that denervation causes elevated transcription of AChRs in extrasynaptical region along the entire length of the muscle fiber (Fontaine et al. 1988; Goldman and Staple 1989; Gundersen et al. 1995), we also verified that the distribution of the AChR is high and diffuse, as would be predicted, for several millimeters from synaptic junctions when myogenin is driven from the HCE mutant promoter compared with the wild-type promoter (Fig. $2 \mathrm{~B}$, bottom) and comparable with the distribution of AChRs in extrasynaptic regions of denervated muscle (Fig. 2B, top and graph). This finding suggests that the HCE-dependent expression pathway for myogenin contributes significantly to the biology of mature muscle by helping to restrict spatiotemporal expression of AChRs to the domain near mature neuromuscular junctions.

\section{Msy3 binds myogHCE}

To search for proteins that interact with myogHCE and in muscle maturation, electrophoretic mobility shift assays (EMSA) were performed with nuclear extracts from skeletal muscle of 3-mo-old mice. Five complexes were seen of which only two (Fig. 3A, arrows) were substantially competed by the unlabeled myogHCE DNA but not by excess mutant HCE DNA. These complexes were not detected in nuclear extracts from younger mice (1-2 wk), and they were no longer detectable after denervation. The $M C K$ enhancer, a known regulatory region active in differentiated skeletal muscle (Lassar et al. 1989), served as a control for the active state of the extracts and 
Figure 1. MyogHCE mediates myogenin RNA levels during postnatal muscle maturation. (A) $\beta$-gal-stained leg muscle of transgenic mice carrying reporter driven by wild-type myogenin promoter (MYOwt) or mutant at myogHCE (MYOmutL). Mouse ages were postnatal 1 wk $(1 \mathrm{w}), 1$ or $5 \mathrm{mo}(1 \mathrm{M}, 5 \mathrm{MM})$, or $1 \mathrm{yr}(1 \mathrm{Y})$. Bar, $400 \mu \mathrm{m}$. (B) Measurements of the expression level of the transgene LacZ by qRT-PCR in leg muscle of transgenic mice carrying the wild-type myogenin promoter (MYOwt) or mutant at myogHCE (MYOmutL, MYOmutT). Mouse ages were postnatal $1 \mathrm{wk}(1 \mathrm{w})$ and 1,2 , or $5 \mathrm{mo}$ (1M, 2MM, 5MM). The expression level is normalized by the endogenous myogenin. Each point derives from RNA isolated from three independent transgenic mice. (C) Measurements of the expression level of the transgene $L a c Z$ by qRT-PCR in control or denervated (D) leg muscles of transgenic mice carrying the wild-type myogenin promoter (MYOwt) or mutant at myogHCE (MYOmutL). Mouse ages were postnatal $1 \mathrm{wk}(1 \mathrm{w})$ and $2 \mathrm{mo}(2 \mathrm{MM})$. The expression level is normalized by the endogenous myogenin. Expression levels of LacZ are reported in Supplemental Figure 4. Each point derives from RNA isolated from three independent transgenic lines. (D) $\beta$-gal-stained leg muscle of 2-mo-old mice, previously electroporated with constructs carrying reporter driven by wildtype myogenin promoter (MYOwt) or mutant at myogHCE (MYOmutL) and nuclear GFP. Bar, $400 \mu \mathrm{m}$. Panels at the left indicates the GFP positive and on the right the LacZ positive nuclei. The graph shows the expression levels of electroporated LacZ transgene and $n G F P$ measured by qRT-PCR.
A

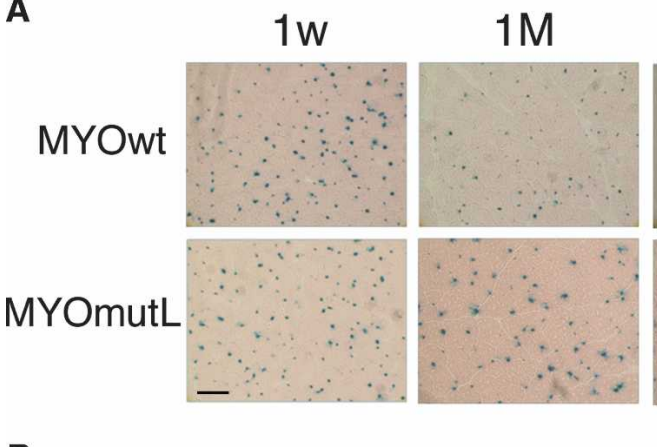

B

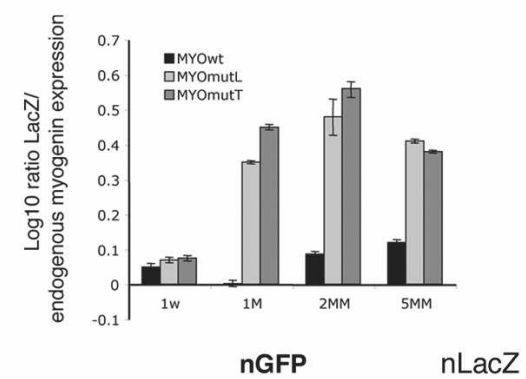

D
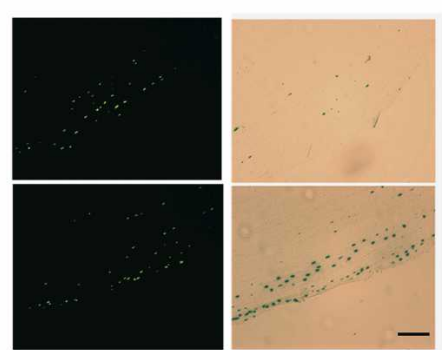

MYOmutL
$5 \mathrm{MM}$
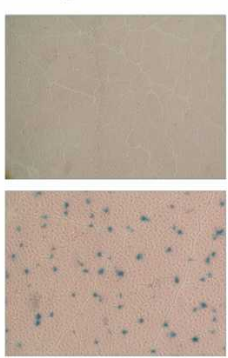

C

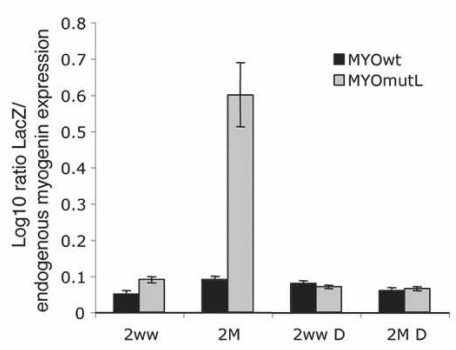

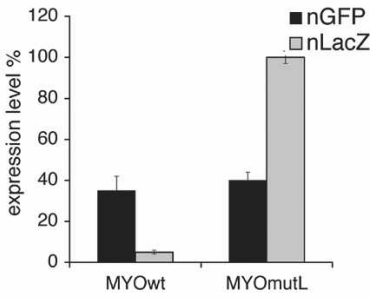

for the EMSA assay. As expected, it produced a consistent binding pattern from the extracts and at all ages, confirming their activity and integrity (Fig. 3B).

To identify proteins that bind the myogHCE, we enriched the protein recognition complex by affinity chromatography from nuclear extracts of skeletal muscle isolated from 3-mo-old mice. We verified that a fraction of this preparation was enriched for the nuclear binding activity of myogHCE by EMSA (Supplemental Fig. 5A). An LC-MSMS mass spectrometry assay (MudPIT as performed in Graumann et al. 2004) revealed that MSY-3, a CSD protein (also known as CsdA, MY1, dpbA, YB-2, ZONAB, or MSY-4), is a significant component identified with $33 \%$ coverage of seven unique peptides. As a group, CSD proteins bind DNA and RNA and they participate in both transcriptional activation and repression in other systems; there is also some evidence that they can affect post-transcriptional regulation /Coles et al. 2000; Kohno et al. 2003). Two forms of MSY-3 mRNA, MSY-3S and MSY-3L, are produced by alternative splicing. Their translated products either possess or lack a 69-amino-acid C-terminal domain. Both isoforms are abundantly expressed in spermatogenic cells, but they are generally at low levels in other adult tissues, except that the MSY-3S isoform is present in heart and abundant in skeletal muscle (Mastrangelo and Kleene 2000). In Figure 6A (below) and Supplemental Figure 6, we show expression of MSY-3 protein at different times during development. The protein was detected in embryonic liver, brain, kidney, heart, and skeletal muscle, while in the adult it was only prominent in testis, heart, and skeletal muscle. Both MSY-3 isoforms are detected in adult muscle fibers, with the MSY-3 short isoform always much more abundant than the MSY-3 long isoform; their expression ratio remains constant during development (Supplemental Fig. 6B,C).

CSD proteins were first identified by their binding to an inverted 5'-CCAAT-3' repeat, but they are considered to generally prefer a range of CT-rich sequences (Shannon et al. 1997). We confirmed the mass spec identification by immunoblotting with the ZONAB antibody (Penes et al. 2005) developed against the canine ortholog of MSY-3 (Supplemental Fig. 5B). We next asked whether MSY-3 has significant innervation-dependent myogHCE-binding activity, as predicted from the way we identified it. EMSA experiments show that this antibody specifically immunocompeted the mobility shift maturation-innervation dependent, whereas addition of 


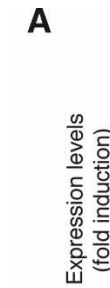

B
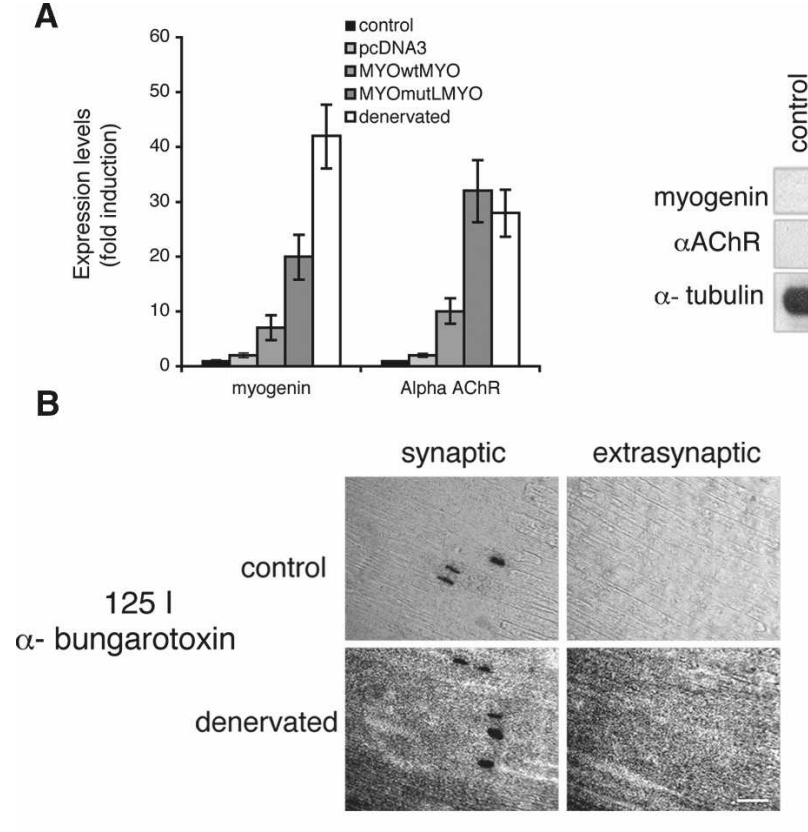

pcDNA3 MYOwtMYO MYOmutLMYO
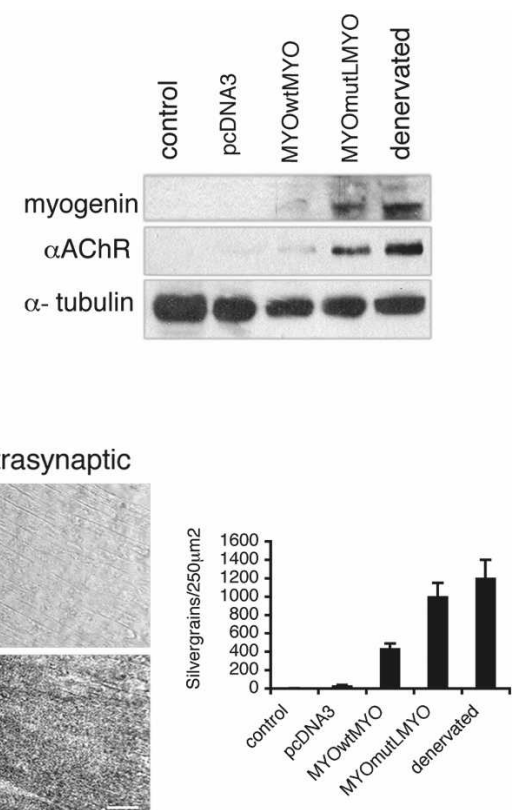

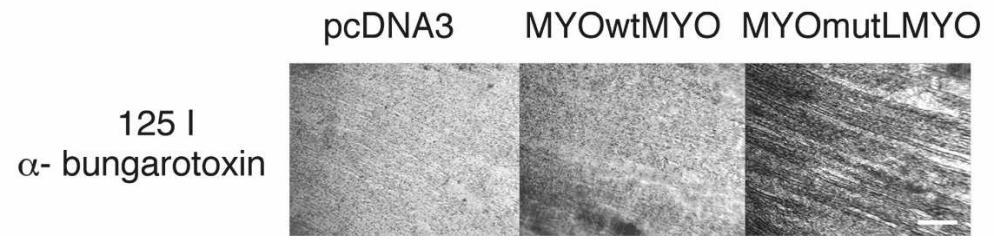

Figure 2. MyogHCE controls AChRs extrasynaptic expression during postnatal muscle maturation. (A) Measurements of the myogenin and AChR $\alpha$ transcripts by qRT-PCR and immunoblot in leg muscle of 2-mo-old mice (control), after $48 \mathrm{~h}$ from denervation (denervated), or electroporated with constructs carrying myogenin cDNA driven by 1.1-kb wild-type myogenin promoter (MYOwtMYO) or mutant at myogHCE (MYOmutLMYO), or an empty pcDNA3 vector. Statistical significance of AChR $\alpha$ transcripts measurements in mutant MYOmutLMYO and in the denervated is $P>0.05$. $(B)$ The images show the AChRs density visualized by autoradiography of longitudinal sections from tibialis anterior muscle innervated (control) or 48-h denervated muscle in synaptic and extrasynaptic fiber segments $(3 \mathrm{~mm}$ from the synaptic junctions) (top) or in extrasynaptic fiber segments $(3 \mathrm{~mm}$ from the synaptic junctions) of tibialis anterior muscle electroporated with an empty pcDNA3 vector, myogenin cDNA driven by the wild-type myogenin promoter (MYOwtMYO) or the mutant at myogHCE (MYOmutLMYO) incubated with 125I- $\alpha$-bungarotoxin (bottom). The sections were subsequently covered with a monolayer of photographic emulsion and after exposure and development silver grains were counted in $250-\mu \mathrm{m}^{2}$ fields along the length of the sections, $3 \mathrm{~mm}$ on each side of the synapse (graph). The areas of high grain density in the left top panels correspond to the endplate region of the fibers. Bar, $400 \mu \mathrm{m}$. a control IgG had no effect, suggesting that the ZONAB binding masks the DNA-binding site of the MSY-3-associated protein complex (Fig. 3C).

Can MSY-3 bind myogHCE in the absence of other muscle nuclear extract components? EMSA was performed with a GST-MSY-3 fusion protein that was incubated with myogHCE oligonucleotides in both doublestranded and single-stranded forms. In this experiment, MSY-3 retarded migration of single-stranded target DNA, in agreement with previous studies that reported that MSY-3 preferentially binds sense ssDNA (Coles et al. 2005). We also observed retarded migration of doublestranded target DNA, even though to a lesser extent quantitatively in comparison with the single strand. Incubation with a mutant oligo, MYOmutL, lacking the myogHCE, revealed that the myogHCE motif is responsible for MSY-3 binding (Fig. 3D).

\section{MSY-3 can inhibit differentiation and requires myogHCE to repress myogenin}

A simple hypothesis based on the lentiviral transgenic results is that a repressor complex binds at the myogHCE during skeletal muscle maturation, and the proteomics screen suggests that MSY-3 is a candidate for mediating all or part of the observed repression. We therefore asked whether added MSY-3 expression could repress myogenin in culture. Multiple, stably transfected MSY-3 C2C12 cell lines were established and analyzed. C2C12 myoblast clones overexpressing MSY-3 protein were compared with control $\mathrm{C} 2 \mathrm{C} 12$ transfected clones, and myogenin was down-regulated at $24-48 \mathrm{~h}$ of differentiation (Fig. 4A) relative to control lines. Their ability to fuse into myotubes and synthesize MHC was also markedly impaired (Fig. 4A, left panels). Effects of extra MSY-3 were not limited to $\mathrm{C} 2 \mathrm{C} 12$, as similar results were obtained for MyoD-converted 10T1/2 cells, in stable and transient transfection formats (Supplemental Fig. 7). We conclude that myogenic differentiation can be disrupted by elevating MSY-3. MSY-3 RNA is expressed in proliferating $\mathrm{C} 2 \mathrm{C} 12$ myoblasts and is normally weakly down-regulated during differentiation in myotubes (Supplemental Fig. 8), so we asked if reducing it further by RNAi could affect differentiation. If MSY-3 normally represses myogenin in myoblasts, then successful MSY-3 knockdown, under the simplest model, could cause premature myogenin expression and/or increase its rate of accumulation, with the net effect of 
A

Figure 3. Innervation-sensitive complexes require the myogHCE element. MSY-3-binding specificity. (A) Protein-binding complexes formed in vitro (arrows), assayed by EMSA, are detected on myogHCE oligo from muscle nuclear extracts of 3-mo-old mice. Labeled MYOwt oligo competed with 10-100-200x excess of cold competitor wild type (MYOwt, lanes 2-4) or mutated (MYOmutL, lanes 5-7), as indicated. Oligo sequences are shown in the box. $(B)$ EMSA complexes (arrows) at myogHCE from nuclear extracts 3-mo-old mice (lane 1) are absent from nuclear extracts of 2 and 1 wk (lanes 2,3) and from nuclear extracts of 3-mo-old mice denervated 24, 48, and $72 \mathrm{~h}$ before (lanes 4-6). Control MCK promoter EMSA (lanes 7-12) shows specific complex from all samples. $(C)$ EMSA of nuclear extracts of muscle of 3-mo-old mice with myogHCE ${ }^{32} \mathrm{P}$-labeled oligonucleotide in the presence of ZONAB antibody (lanes 2,3, 1-5 $\mu \mathrm{g}$ ) or control IgG (lane 4, $5 \mu \mathrm{g}$ ). Arrows indicate the specific, asterisks indicate the nonspecific, and arrowheads the immunocompeted mobility shift maturation-innervation dependent protein complexes. (D) EMSA assay of GST and GST-MSY-3 fusion protein with myogHCE ${ }^{32} \mathrm{P}$-labeled oligonucleotide in single strand forms (lanes 1,2 sense; lanes 4,5 antisense) and in a double strand (lanes 7,8) and GSTMSY-3 protein with mutant myogHCE ${ }^{32} \mathrm{P}$-labeled oligonucleotides MYOmutL in single strand forms (lane 3, sense; lane 4, antisense) and in a double strand form (lane 9).

accelerating myogenin-regulated differentiation phenotypes. When a pool of four siRNA oligos targeting both MSY-3 isoforms was used in C2C12 cultures, strong activation (10- to 14-fold) of myogenin and its target genes, $\alpha$ AChR and MCK, was observed (Fig. 4B). A weaker up-regulation (approximately threefold) of MyoD was also observed. By immunostaining for myogenin protein in cell cultures treated with MSY-3 or control siRNAs, precocious positive cells were seen under proliferationpromoting, myoblast conditions (growth medium [GM]), where myogenin is normally absent (Fig. 4B, right panels). Furthermore, we could rescue the differentiation capacity of a C2C12 MSY-3-overexpressing clone (clone 5) by MSY-3 RNAi, whose effect was monitored as demonstrated by measuring MHC (Supplemental Fig. 9). Deletion of the CSD strongly abrogates the ability of an MSY-3 expression construct to repress myogenin levels or myogenesis and induced a hypertrophic phenotype, which is absent in other mutants or in the control (Fig. 4C). Mutation of the MSY-3 protein in its putative DNAbinding domains showed that deletion of the CSD also prevented binding to both single-sense (SS) and doublestranded (DS) myoHCE DNA (Supplemental Fig. 10). These results suggest that DNA binding mediated through the CSD is required for myogenic inhibition.

The gain-of-function and loss-of-function experiments described above suggest that MSY-3 behaves as a repressor of myogenesis. To further probe the mechanism, we asked whether MSY-3 acts through the myogHCE motif in $\mathrm{C} 2 \mathrm{C} 12$ differentiation. To do this, we made multi-
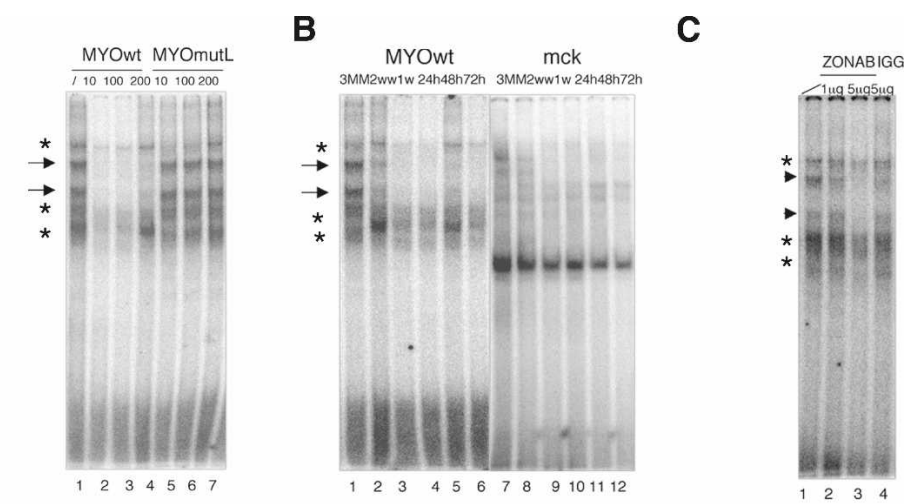

MYOwt AACGTCTTGATGTGCAGCAACAGCTTAGAGGGG MYOmutL . . . - GACCGAGTCGT TA ACTAAT . . . . . . . . .

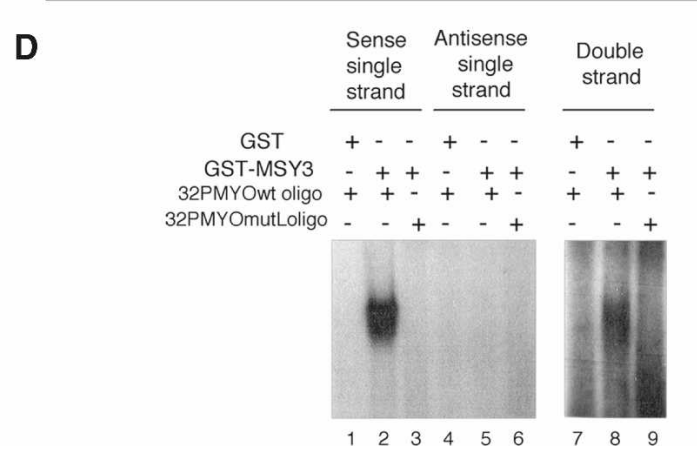

clonal transformant pools carrying a stably transfected nuclear $L a c Z$ reporter driven by the 1.1-kb wild-type myogenin promoter or by either of its two myogHCE mutated versions (MYOmutL and MYOmutT, the same mutant versions used in the transgenic analysis); then we transfected each reporter pool with a construct that drives expression of MSY-3 (Msy) or with a mock vector (pc). LacZ reporter RNA was quantified by qRT-PCR throughout differentiation. In the absence of added MSY3 , mutated and wild-type myogenin constructs increased similarly (Fig. 5A). With excess MSY-3, a greater increase was observed from mutant promoters (MsymutL or MsymutT) versus wild type (MsywtM) in late differentiation $(96 \mathrm{~h})$. This recapitulates the effect of MSY-3 overexpression on endogenous myogenin. These data demonstrate the ability of MSY-3 to regulate expression from the myogenin promoter but also show that the effect is context-dependent and becomes significant later in differentiation when MyoD levels are diminished and its binding is diminished relative to earlier times (Supplemental Fig. 11). This suggests that mutual exclusivity between MSY-3 and MyoD could mediate the choice between an open and induced myogenin promoter and a repressed one.

To determine if MSY-3 associates directly with the myogenin locus in vivo we performed chromatin immunoprecipitation (ChIP) from proliferating and differentiating $\mathrm{C} 2 \mathrm{C} 12$ myoblasts, using the $\mathrm{ZONAB}$ antiserum against the MSY-3 protein. Chromatin immunoprecipitated from proliferating $\mathrm{C} 2 \mathrm{C} 12$ myoblasts with $\mathrm{ZONAB}$ 
A
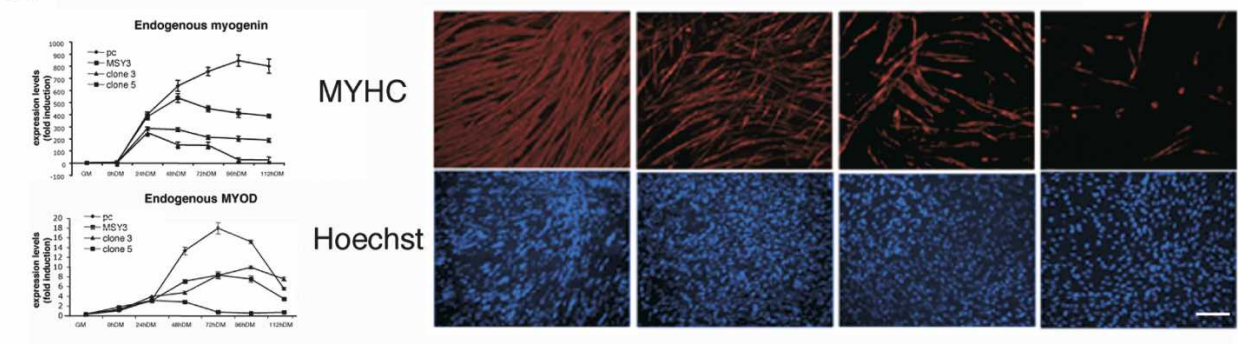

pcDNA3

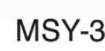

clone3

clone5
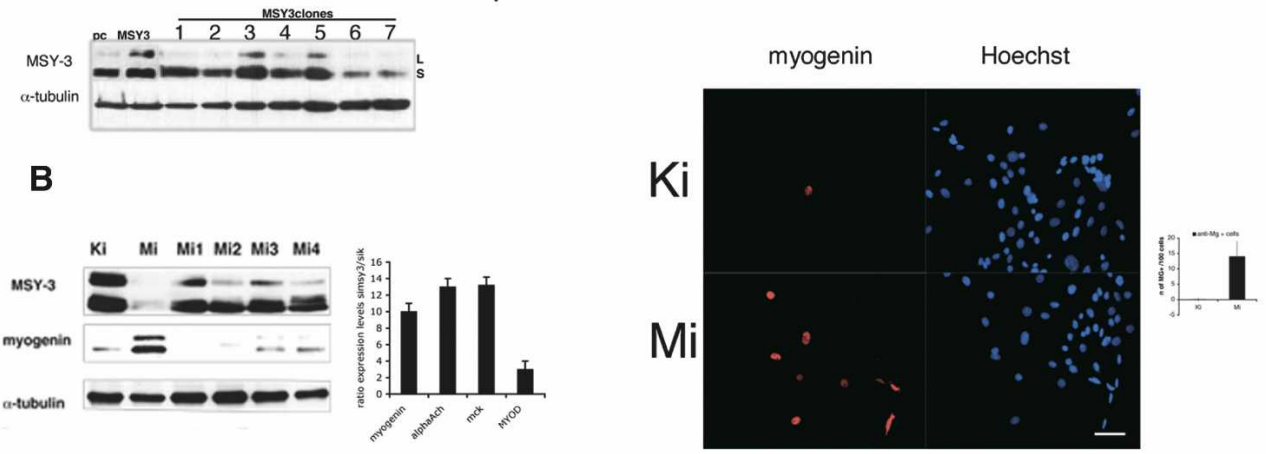

в

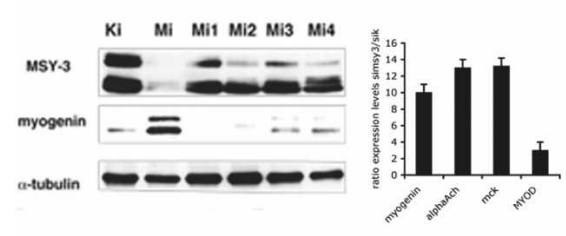

C

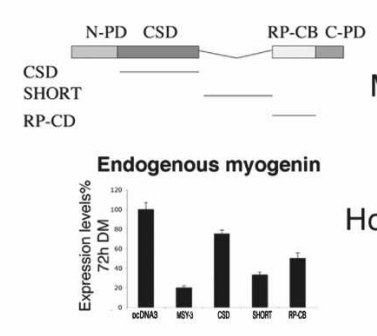

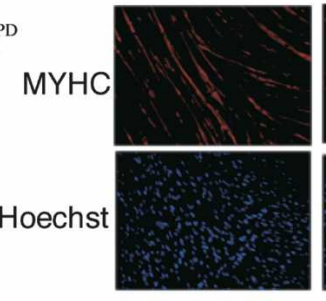

MSY-3

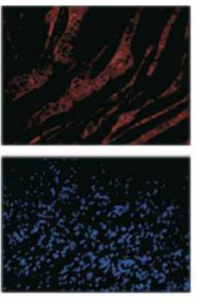

CSD

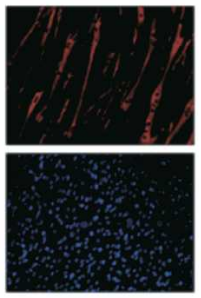

SHORT

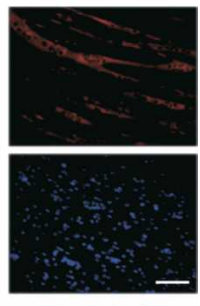

RP-CB

Figure 4. Evidence MSY-3 inhibits myogenesis. (A) MSY-3 transfections of C2C12 myogenic cells. Expression during myogenic differentiation of endogenous myogenin and MyoD measured by qRT-PCR of mock-transfected (pc) and a MSY-3-transfected (MSY-3) C2C12 multiclonal populations, and two MSY-3-transfected clones (3 and 5). Immunoblot of extracts from MSY-3-overexpressing C2C12 clones blotted with ZONAB (MSY-3 Ab) and $\alpha$-tubulin control. Two MSY-3 isoforms, short (S) and long (L). Myosin heavy chain $\mathrm{Ab}$ marks differentiation (left) and Hoechst (right) stains of nuclei of the same $\mathrm{C} 2 \mathrm{C} 12$ multiclone populations and clones 3, $5 \mathrm{at} 72 \mathrm{~h}$ in DM (full differentiation). Bar, $400 \mu \mathrm{m}$. (B) Effects of MSY-3 knockdown by siRNA. The immunoblot shows the two isoforms of MSY-3 protein in extracts from C2C12 in GM, transfected with a pool of four interfering oligos (MiSmart) against MSY-3; or single oligos (Mi1, Mi2, Mi3, Mi4), compared with control oligos (KiSmart). Levels of myogenin are reported and normalized relative to $\alpha$-tubulin. Left graph shows the ratio of myogenin, MyoD, $\alpha$ AChR subunit, and MCK between the C2C12 in GM blocked with MSY-3 siRNA (MiSmart) or with control siRNAs (KiSmart). GAPDH was used to normalize. Anti myogenin Ab (left panel) and Hoechst (right) staining of control siRNA (KiSmart) or MSY-3 siRNA (MiSmart). Bar, $200 \mu \mathrm{m}$. Graph quantifies myogenin positive C2C12 nuclei in growth medium. (C) Mutational analysis of MSY-3 protein. C2C12-transformed cell lines overexpressing mutated forms of HA-tagged MSY-3 protein were derived. In Supplemental Figure 10A expression of MSY-3-HA tagged in MSY-3 wild-type and mutant polyclone populations is shown. Domain structure of MSY-3 protein and mutated forms derived N-PD (N-terminal proline-rich domain), CSD (cold-shock domain), SHORT (splicing alternative domain), RP-CB (arginine- and proline-rich conserved domain), C-PD (C-terminal praline-rich domain). The graph indicates the expression level of endogenous myogenin in the clones overexpressing the wild-type MSY-3 protein, and the mutated forms in the CSD, SHORT, and the RP-CB domain. Myosin heavy chain Ab marks differentiation (top) and Hoechst (bottom) stains nuclei of the same C2C12 multiclone populations at $72 \mathrm{~h}$ in DM (full differentiation). Bar, $400 \mu \mathrm{m}$.

shows enrichment of the myogenin promoter that is comparable with the enrichment seen with an antiMyoD antibody in differentiating cells (Fig. 5B). Because it was previously proposed that MSY-3 directly represses the $\delta$ cholinergic subunit gene (Sapru et al. 1996), we extended the ChIP analysis to the AChR site. No significant enrichment of the AChR activity-dependent en- hancer was observed, although the myogenin promoter was again enriched (Supplemental Fig. 12). MSY-3 requires an intact myogHCE sequence to bind at the myogenin promoter in cells, as shown by ChIP analysis of $\mathrm{C} 2 \mathrm{C} 12$ multiclone populations carrying the LacZ transgene under the myogenin promoter wild type and mutated (Fig. 5C). Furthermore, Figure 5D shows that when 
Figure 5. MSY-3 requires myoHCE to repress myogenin reporter and binds in vivo at myogenin promoter. (A) Measurements of $L a c Z$ transgene reporter by qRT-PCR in $\mathrm{C} 2 \mathrm{C} 12$ clones. $L a c Z$ is under the control of the wild-type myogenin promoter (pcMwt, MsyMwt) or mutated variants (pcmutL, MsymutL pcmutT, MsymutT) in mock-transfected (pc) and MSY-3-overexpressing (Msy) C2C12 cells. Expression level reported as log ratio of LacZ mutated/wild type, normalized by endogenous myogenin. Neomycin and puromycin expression markers used to check that transgene incorporation levels were similar. $(B)$ ChIP analysis for MSY-3 and MyoD. Control IgG, MSY-3, and MyoD antibodies were used individually to enrich fixed chromatin from undifferentiated (GM) or differentiated (DM) $\mathrm{C} 2 \mathrm{C} 12$ cells. qRT-PCR is used to quantify sequences from the myogenin promoter and the negative control $\mathrm{IgH}$ enhancer. $(\mathrm{C})$ Chromatin from multiclone populations carrying $\mathrm{LacZ}$ under MYO and MYOL promoter control growth in GM. qRT-PCR used to quantify for the ectopic myogenin promoterLacZ fragment (left) and the endogenous myogenin promoter (right). $(D)$ Chromatin from undifferentiated $\mathrm{C} 2 \mathrm{C} 12$ transfected with the pool of four interfering oligos (Mi) that knocks down MSY-3 or control oligos (Ki). Figure displays results representative from three independent ChIP experiments.

MSY-3 is knocked down in proliferating C2C12 cells, ChIP enrichment of the myogenin promoter is greatly reduced, compared with control cultures. The myogenin locus is also detectably occupied by MyoD under these conditions of MSY-3 knockdown, suggesting that MyoD can bind the myogenin promoter prematurely under myoblast growth conditions if, and only if, MSY-3 is reduced. This suggests that MSY-3 might act, at least in part, by competing with MyoD for occupancy of myogenin promoter.

\section{MSY-3 in adult muscle innervation}

MSY-3 repression of myogenin in maturing and mature muscle suggests that it may be directly involved in regulating muscle responses to innervation and denervation. Figure 6A shows that MSY-3 protein levels increases during muscle maturation but start to decrease $6 \mathrm{~h}$ after denervation. This profile is the opposite of those for myogenin, MyoD, and AChR $\alpha$ subunit genes. We experimentally forced precocious MSY-3 expression in neonatal muscle in vivo by electroporating quadriceps and tibialis anterior muscles of 9-d-old mice with a MSY-3 expression vector. Myogenin and $\alpha$ AChR RNA expression, $5 \mathrm{~d}$ after electroporation, were reduced $60 \%$ compared with levels in muscle receiving the control
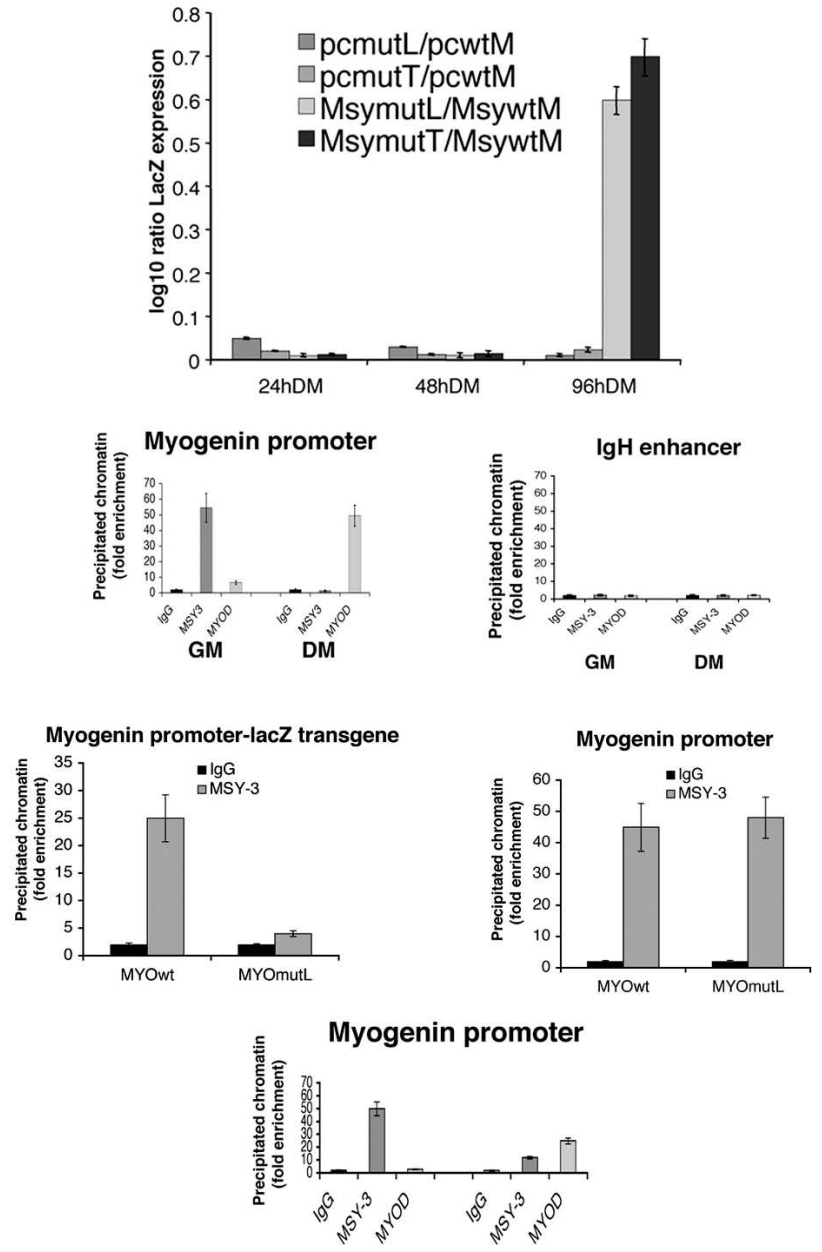

construct (Fig. 6B). This is consistent with MSY-3 acting as a negative regulator of myogenin in the animal. When an MSY-3-expressing construct was electroporated into denervated muscle, reduction was observed in transcripts normally induced upon denervation, including myogenin, MyoD, and $\alpha$ AChR. In contrast, no change was detected in $\varepsilon \mathrm{AChR}$ or muscle creatin kinase, genes whose RNA is known to remain constant after denervation (Fig. 6C). We also attempted to reduce MSY-3 in vivo by electroporating RNA-interfering oligos in the tibialis anterior muscle of 2-mo-old mice; an increase of $\sim 50 \%$ in myogenin and AChR $\alpha$ transcript levels followed, while there was no detectable elevation of the innervation insensitive control gene, MCK (Fig. 6D, left). Furthermore, the distribution of the AChR became diffused over several millimeters from the synaptic junctions in regions of the muscle where MSY-3 is knocked out (Fig. 6D, right). These data are consistent with a role for MSY-3 in repressing myogenin expression during muscle innervation.

MSY-3 and Pbx occupy myogHCE region during muscle maturation

An elegant study by Tapscott and colleagues (Berkes et al. 2004) showed that the sequence domain located at 
A

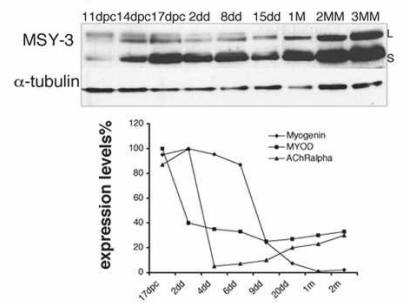

B

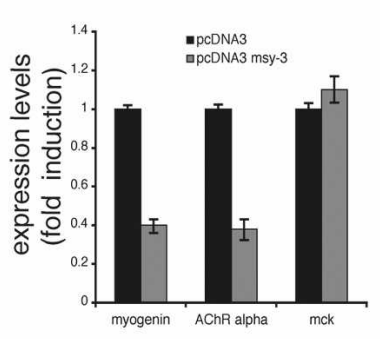

D

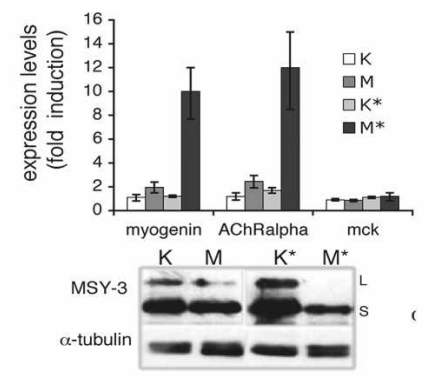

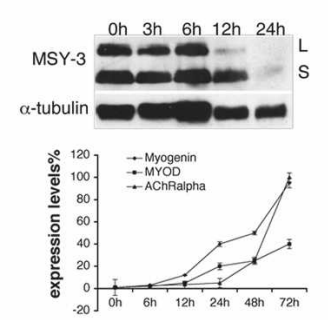

C

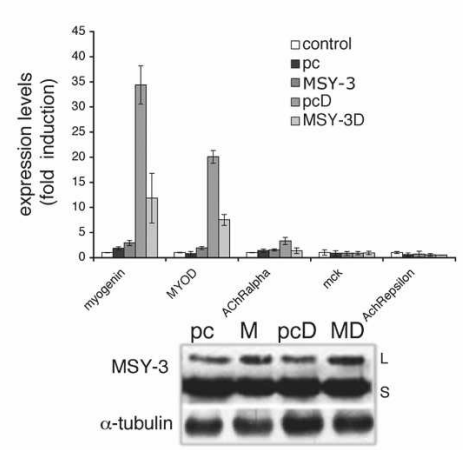

Ki

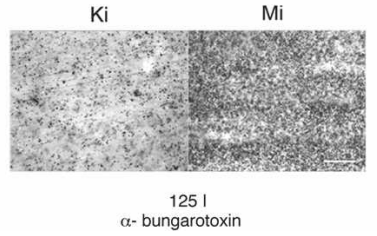

Figure 6. MSY-3 in adult muscle innervation. (A) Expression of MSY-3 protein and myogenin, MyoD and AChR $\alpha$ subunit RNA in muscle at different times of maturation (immunoblot and graph, left) and after denervation by sciatic nerve transection (immunoblot and graph, right). $(B)$ Expression of myogenin and AChR $\alpha$ subunit genes after electroporation in tibialis anterior and quadriceps muscle of young mice (14 d postnatal) of the control (pcDNA3) and MSY-3 (pcDNA3-MSY-3) constructs evaluated by qRT-PCR. $(C)$ Expression of myogenin, MyoD, AChR $\alpha$ subunit, MCK, and AChR $\varepsilon$ in adult ( $2 \mathrm{mo}$ ) tibialis anterior muscle (control) electroporated with pcDNA3 (pc); electroporated with MSY-3 (MSY-3); electroporated with the same constructs and later denervated by sciatic nerve transection $/ \mathrm{pcD}$ and MSY-3D). Expression was evaluated by qRT-PCR and immunoblot (MSY-3). ( $D$, left) Expression of myogenin, AChR $\alpha$ subunit, and MCK in tibialis anterior muscle of adult mice $(2 \mathrm{mo}$ ) elecroporated with a pool of four interfering oligos $(\mathrm{Mi})$, which blocks MSY-3 or with control oligos (Ki), coinjected with a nuclear GFP-expressing plasmid $\left(\mathrm{Mi}^{\star}\right.$ and $\left.\mathrm{Ki}^{\star}\right)$ in order to dissect only the injected area and evaluated by qRT-PCR. The immunoblot measures MSY-3 expression. (Right) AChRs density in extrasynaptic regions $(3 \mathrm{~mm}$ from synaptic junctions) visualized by autoradiography of longitudinal sections from $\mathrm{Ki}^{\star}$ or $\mathrm{Mi}^{\star}$ electroporated tibialis anterior muscle of adult mice (2 mo old) and incubated with $125 \mathrm{I}$ - $\alpha$-bungarotoxin. Bar, $200 \mu \mathrm{m}$.
-98 of the mouse myogenin promoter is required by MyoD for a stable binding to the myogenin promoter region. They showed that this binding by MyoD is important for a chromatin opening function that normally precedes myogenin activation, and that it requires an interaction with the homeodomain protein $\mathrm{Pbx}$, which binds at a site adjacent to MyoD. That led us to hypothesize that Pbx might also interact with MSY-3 in the innervation-stimulated repression function. The expression level of two of the three isoforms of $\mathrm{Pbx}(\mathrm{Pbx} 1$ and $\mathrm{Pbx} 3$ ) suggests a possible role in adult muscle innervation (Fig. 7A). Both isoforms reach their peaks of expression around day 20 of postnatal life when normal innervation is prominent and when myogenin is aggressively down-regulated (Fig. 6A). To determine if $\mathrm{Pbx}$ is bound at the myogenin locus in muscle, and to compare its occupancy with that of MSY-3 and MyoD during maturation, we performed ChIP experiments. Pbx ChIP displayed greater enrichment from muscle of 2-wk-old and 2-moold mice, compared with muscle from younger mice $(2 \mathrm{~d}$ and $1 \mathrm{wk}$ ), as did MSY-3 (Fig. 7B). To determine if Pbx protein and MSY-3 can bind to the same fragment of the myogenin promoter simultaneously, we performed a se- quential RE-Chip assay; by immunoprecipitating with the Pbx antisera the chromatin first immunoprecipitated with the anti-MSY-3 antibody. We observed an enrichment of the immunoprecipited chromatin when the muscle had been innervated recently $(2 \mathrm{wk})$, suggesting that the association between Pbx and MSY-3 at the myogenin locus is simultaneous in vivo in recently innervated muscle (Fig. 7C). MSY-3 requires an intact myogHCE sequence to bind at the myogenin promoter in vivo, as shown by ChIP analysis of muscle from LacZ transgenic mouse, lines MYOwt and MYOmutL (Supplemental Fig. 13).

To test the possibility that the myogHCE-like conserved element located $35 \mathrm{~kb}$ upstream of $M y o D$ (chr7:42,517,839-42,517,855, UCSC $\mathrm{mm} 7$ ) might also bind MSY-3 in vivo, ChIP assays were performed. MSY-3 enrichment was detected at the upstream $M y o D$ domain, similar to results for the $-1.1-\mathrm{kb}$ myogenin region, although the two loci differed in extent of occupancy by MyoD (Fig. 7B, bottom), which might be related to the presence of a canonical E-box in the HCErelated MyoD sequence versus a noncanonical one in myogenin. 
A

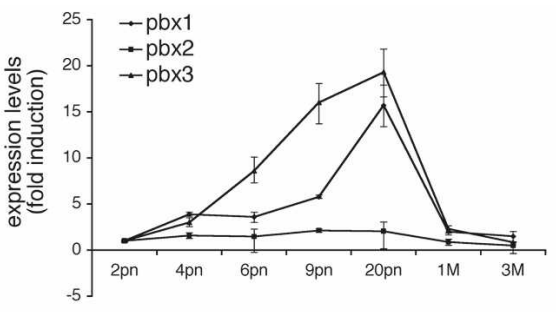

B

Figure 7. MSY-3 and Pbx binding to the myogHCE region in vivo. (A) Expression of three isoforms of $\mathrm{Pbx}$ in muscle at different times of maturation are evaluated by qRT-PCR. (B) ChIP analysis with control IgG, MSY-3, MyoD, and Pbx antibodies on chromatin from tibialis anterior muscle of young ( $2 \mathrm{dd}, 1 \mathrm{w}$, and $2 \mathrm{ww})$; adult $(2 \mathrm{MM})$; or denervated (2MMD) mice. Following immunoprecipitation, DNA enrichment was measured by qRT-PCR for myogenin promoter (top left), control IgH enhancer (top right), or -35-kb MyoD enhancer (bottom). (C) A sequential ChIP (RE-ChIP) experiment. Chromatin immunoprecipitated with MSY-3 was reincubated with anti $\mathrm{Pbx}$ (Pbx/MSY-3) or vice-versa (MSY-3 then $\mathrm{Pbx} \mid$. Results shown are representative of three independent experiments.

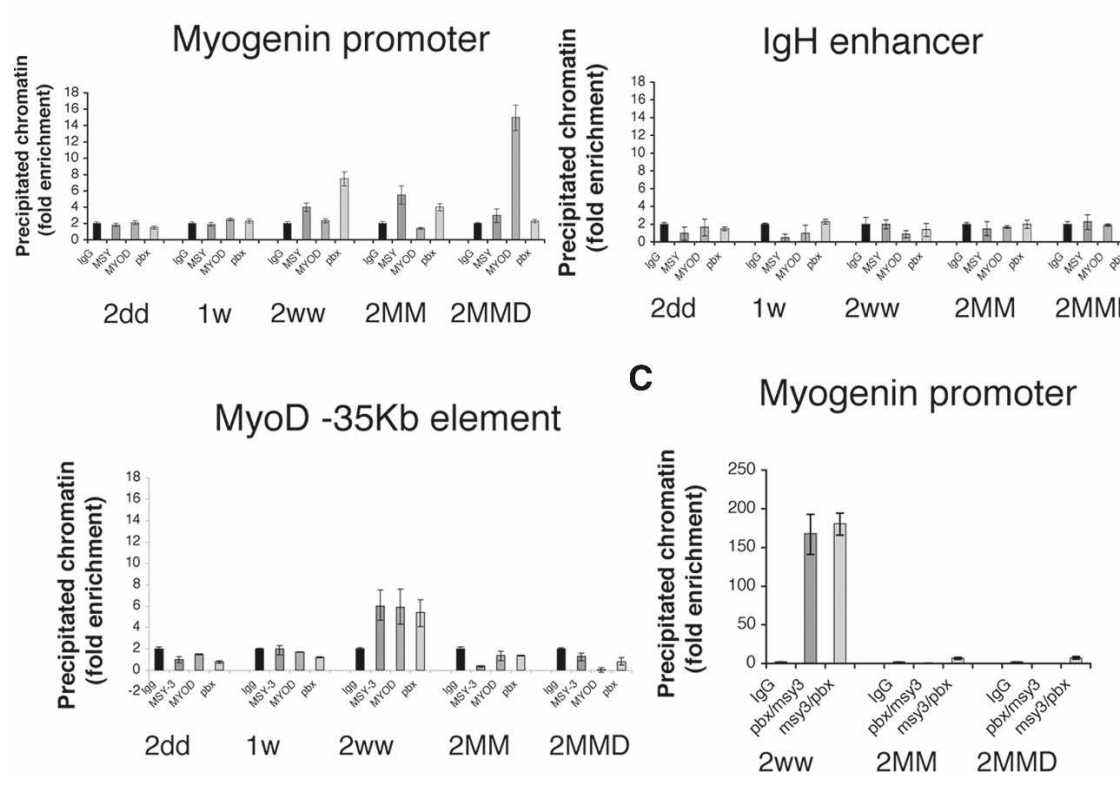

\section{Discussion}

The central findings of this study spring from the observation that the myogHCE conserved cis element is critical for transcriptional repression of myogenin in postnatal muscle maturation. We then identified MSY-3 as a trans-acting factor that binds to the myogHCE in vivo and in vitro and can mediate repression. Regulation of myogenin expression has been of longstanding interest because myogenin plays a critical and unique role in the muscle regulatory network as a direct positive-acting transcription factor for hundreds of skeletal muscle differentiation genes, including autoregulation and positive cross-talk with other transcription factors of the core myogenin gene regulatory network. But myogenin expression does not continue at high levels in normal adult muscle, and MRF4 ultimately becomes the major MRF family member. The down-regulation of myogenin in mature muscle also has a spatial dimension related to innervation. Thus, AChRs, MusK, rapsyn, and other proteins necessary for AChR subunit clustering at neuromuscular synapses are especially myogenin sensitive, and their expression is broadly repressed in most extrajunctional myonuclei in response to muscle innervation. Levels of myogenin and MyoD expression appear to be crucial in this process (Blais et al. 2005; Macpherson et al. 2006).

Our findings suggest that the myogHCE/MSY-3 regulatory node plays a key role in the spatiotemporal repres- sion of myogenin in mature, innervated muscle. We do not yet know exactly how MSY-3, which apparently occupies the myogHCE motif in collaboration with $\mathrm{Pbx}$, mediates repression. One possibility is suggested by recent studies that point to HDAC activity at the myogenin locus (Mejat et al. 2005; Tang and Goldman 2006). HDAC recruitment has been associated with MEF2 and Six factors in those studies, and both have conserved sequence motifs located between the myogHCE and the myogenin promoter. The fact that we observed small, but nevertheless statistically significant, repression in our assays of myogHCE mutant transgene activity is consistent with the idea that myogenin repression has multiple contributing elements, and that MEF2/HDAC and Six/Dach/HDAC are likely to be responsible for this additional repression activity. However the effects that remained after the HCE was eliminated were quantitatively quite minor in our study, and this is consistent with the observation that HDAC inhibition by trichostatin in mature innervated muscle failed to relieve myogenin repression (Tang and Goldman 2006). It is attractive to think that the three cis-trans systems together ensure repression, and there might be significant interplay between MSY-3 and HDAC systems.

In the $\mathrm{C} 2 \mathrm{C} 12$ cell culture model of muscle differentiation, which precedes the maturation events discussed above, the myogHCE cis element can act positively rather than negatively, and it does so by binding MyoD 
and Pbx (Berkes et al. 2004). MSY-3 is expressed in C2 cells, and this raised the question of whether it has the capacity to repress premature differentiation in myoblasts or it could alter the onset of differentiation. In $\mathrm{C} 2 \mathrm{C} 12$ cells, where the chromatin opening function was first discovered, we found we could alter myogenin expression (and its positive regulatory effects on differentiation), by elevating or reducing MSY-3 relative to its normal levels. The simplest interpretation of these results is that, in the presence of both MyoD and MSY-3, the HCE can act in either a positive or negative manner, and the choice depends on the relative levels and activities of the positive (MyoD) or negative (MSY-3) factors, both of which seem to collaborate with PBX. This appears to be one instance of a more general regulatory strategy that features dual function cis-regulatory elements. Thus, they normally switch their regulatory sign from positive, which dominates during muscle differentiation (MEF2, Six-1, MyoD/PBX, in association with their coactivators), to negative, which dominates during muscle maturation and innervation (MEF2 and Six/Dach in association with HDACs and MSY-3/PBX).

When we studied the effects of the myogHCE on expression in lentiviral transgenic mice, we did not see an obvious phenotype corresponding to the activating one already described in $\mathrm{C} 2 \mathrm{C} 12$ cells (Berkes et al. 2004). We saw apparently normal $L a c Z$ reporter expression from the mutated contructs at sites in the embryos where new myogenic differentiation is occurring, such as young caudal somites and limb buds. These are domains where one might have expected to see effects from a failure of chromatin opening as a failure to turn on at the same time as wild type, or to activate to the same extent. Failure to see a clear effect of this kind at first seemed surprising, but the LacZ staining assay we used may not be quantitative enough to detect lowered myogenin activation on a cell-by-cell basis, nor sensitive enough to pick up a more gradual up-regulation. In addition, the lentiviral vector could be providing its own chromatin open domain information that accomplishes for the construct what the myogHCE normally does in the native myogenin locus.

During postnatal development, both lentiviral and conventional transgenic assay systems revealed a robust myogHCE effect on postnatal myogenin expression. The fact that myogenin is strongly repressed postnatally is generally compatible with the fact that a conditional knockout, which takes its full effect in this time period, is not lethal and mainly alters adult animal size (Knapp et al. 2006). That study does, however, raise the question of whether there is normally a time during or after muscle maturation when myogenin ceases to be important to specific endogenous target genes expressed at junctional myonuclei, and what regulators take over that job when myogenin is knocked out.

Previous studies showed there are some additional cisacting myogenin regulatory regions that are responsive to electrical activity in postnatal life, positioned upstream (i.e., between -1102 and -1565 ) relative to the region in our constructs (Gibney and Buonanno 1995).
To our knowledge, the cis elements in that more distal region have not been more finely localized. We did not find a second sequence motif similar to myogHCE over entire myogenin locus, but it is possible and even likely that additional cis-acting modules contribute to nervemediated myogenin regulation. The possibility that MSY-3 might directly repress additional genes that are differentially expressed in extrajunctional myonuclei synaptic ones is an idea with some appeal, since it would be a way to provide regulatory robustness and/or finetuning. A specific role for MSY-3 in regulating the $A C h R$ $\delta$ subunit gene would fit this model and is supported by a prior observation of in vitro binding by MSY-3 (Sapru et al. 1996). However, that study did not demonstrate in vivo occupancy of the $A C h R$ enhancer, so we performed ChIP experiments. No interaction was detected under conditions that gave positive enrichment for MSY-3 at the myogenin HCE. We do not know if this is a technical experimental difference-perhaps revolving around the sensitivity of ChIP enrichment-or a biological difference between occupancy at myogenin and the possible occupancy at $A C h R \delta$. In any case, the fact that AChR expression is disregulated by ectopic myogenin in our experiments argues that the MSY-3 normally present in the system is not sufficient to keep AChRs fully repressed on its own.

MSY-3 appears to be a versatile factor, and it is likely to have regulatory targets other than myogenin in our system. They need not all be repressive. For example, MSY-3 has been reported as a positive acting regulator of genes associated with cell cycle progression (Sourisseau et al. 2006). In particular, our observation in myogenesis that MSY-3 overexpression induces some muscle apparent hypertrophy is consistent with the idea that it has multiple effects, some of which might be independent of DNA binding, as suggested by the CSD mutant phenotype.

Although it was not a focus of this study, we found some initial evidence that $\mathrm{MyoD}$ might also be directly affected by MSY-3. A match for the myogHCE PSFM was discovered within a larger, unstudied conserved feature located at $\sim 35 \mathrm{~kb}$ upstream of $M y o D$ transcription initiation in mouse, and $10 \mathrm{~kb}$ upstream of the major "core" $M y o D$ enhancer (Goldhamer et al. 1995). By ChIP criteria, MSY-3 binds to the $-35-\mathrm{kb}$ region in vivo. The possibility that both MyoD and myogenin are affected by the same repressor complex during muscle innervation, recognizable by a similar cis motif, has appealing simplicity and would help to explain the strong phenotypic effects of MSY-3 ectopic expression and knockdown.

Multiple lines of evidence presented here support the view that myogHCE is a critical cis element for repression of myogenin. The myogHCE is composed of partly overlapping binding sites for MyoD, MSY-3, and PBX, and the partial overlap of binding sites provides a plausible explanation for strong conservation over great distances that separate mammals and fish. It also could provide a way to make the regulatory states mutually exclusive, and therefore switch-like. However, the preferential conservation does not correspond, in this 
case, to biochemical optimality of the individual binding motifs for the individual factors. Quite the opposite is true, since none of the sites in myogHCE would even be identified by sequence inspection as motifs for MyoD, MSY-3, or Pbx. Multiple weak individual binding motifs could, however, offer special advantages for regulating cell state transitions, which we understand myogenin does. Thus, individually weak binding sites, stably occupied only when all members of a multifactor complex are present at high levels and in the right modification states, should prevent erroneous changes of state; yet they would also be sensitive, on a rapid time scale, to big changes in any one component, which would readily destabilize the entire complex. For myogenin, the paths would be from repressed in myoblasts to active in early differentiation; from active in early differentiation to a repressed state in innervated mature myofibers; and still later, from repressed in healthy innervated myofibers to reactivated upon denervation or, perhaps, to degeneration, which has not yet been explored. Although evolution of the myogHCE and its surrounding locus cannot be tested directly, the impact of "optimizing" individual motifs, and their pairwise combinations, in the myogHCE should be experimentally accessible using assay systems developed here for repression and previously by Berkes et al. (2004) for activation.

The three cis-regulatory themes proposed for myogenin-(1) highly conserved biochemical suboptimality in the HCE, for sensitivity and specificity; (2) conserved binding site overlap for exclusivity of action between MyoD and MSY-3, in the HCE; and (3) individual cis elements able to bind complexes of opposing activites, according to cell context (Six, MEF2, as well as HCE)can now be searched for in other systems to see if they are general and if they are especially used for regulators of cell state.

\section{Materials and methods}

Comparative genomics

Procedures are described in the Supplemental Material.

\section{Cell, transfections, and tissue cultures}

293T, C2C12, and C3H10T1/2 cells were from American Type Culture Collection. C2C12 cells were cultured in DMEM supplemented with $20 \%$ FBS (GM) and induced to differentiate with DMEM supplemented with $2 \%$ horse serum (DM, differentiation medium). $293 \mathrm{~T}$ and $10 \mathrm{~T} 1 / 2$ were cultured in $10 \%$ FBS. Transfections and stable clones were generated as described in the Supplemetal Material. Muscle fiber cultures were performed as described in Cornelison and Wold (1997).

\section{Plasmids and lentiviral constructs}

Constructs and the cloning details are described in the Supplemental Material. For the lentiviral transgenesis we generated the wild-type myogenin promoter lentiviral vector pFMYOwt $L a c Z$ and the mutated versions pFMYOmutLLacZ and pFMYOmutTLacZ as described in Supplemental Figure 2.

\section{Lentiviral and conventional transgenesis}

Injection of lentiviruses into mouse embryos was performed according to Lois et al. (2002). Details of conventional transgenesis and lentiviral tritation and transgenics analysis are described in the Supplemental Material and in Supplemental Table S2.

\section{Detection of acetylcholine receptors}

The density of AChRs in muscle was determined as described by Gundersen et al. (1995), with the exception that longitudinal sections of tibialis anterior muscle were incubated with 125 $\mathrm{I}-\alpha$-bungarotoxin and then processed as described.

\section{Tandem affinity purification MudPIT}

MudPIT mass spec analysis of proteins retrieved from affinity enrichment by binding to myogHCE DNA was performed essentially as described in Graumann et al. (2004). Samples were proteolytically digested and analyzed by multidimensional chromatography in-line with a Deca XP ion trap mass spectrometer (ThermoElectron). Samples were released stepwise from the strong cation exchanger phase of the triphasic capillary columns as reported previously (Graumann et al. 2004).

\section{In vivo electroporation}

Tibialis anterior and quadriceps muscles were electroporated as previously described with minor modifications (Protocol 2, Martinenghi et al. 2002). Briefly, mice were anesthetized by avertin injection, and expression vectors were injected with a 0.5 - $\mathrm{m}$ insulin syringe through a 27 -gauge needle into quadriceps and tibialis anterior muscles of 9-d CD1 male mice (20 $\mu \mathrm{g}$ of each plasmid in a costant volume of $20 \mu \mathrm{L}$ of PBS) and into tibialis anterior muscles of 2 -mo-old CD1 female mice $50 \mu \mathrm{g}$ of each plasmid in $50 \mu \mathrm{L}$ of PBS). A pair of stainless steel plates was then applied over the muscle to encompass the injection area. Current was delivered 5 to $10 \mathrm{~min}$ after DNA injection as a constant current, square-wave pulse with a digital Stimulator (Panlab 3100, Biological Instruments) and checked by a digital oscilloscope (Tektronix TDS210). The characteristics of the electric field applied were $200 \mathrm{~V} / \mathrm{cm}, 20 \mathrm{msec}$ amplitude, $1 \mathrm{~Hz}$, eight consecutive. In denervation experiments the tibialis anterior muscles were denervated by sciatic nerve transection $8 \mathrm{~d}$ later than electroporation and tissue was processed for RNA extraction $24 \mathrm{~h}$ later than denervation. In each electroporation experiment, $5 \mu \mathrm{g}$ of a lentiviral vector expressing nuclear GFP is coinjected and, when indicated by ${ }^{*}$, the GFP postitive area was dissected to restrict the injection area for successive analysis.

RNAi

The duplex siRNA sequences used in RNAi are Mil, CGACG UCCCUACAACUAUAUU; Mi2, CGUCAGAAAUGGAUAU GGAUU; Mi3, AAACAACCCACGCAAGUAUUU; Mi4, CA ACGCUGUUUCACAAGAUUU.

Methods are described in the Supplemental Material.

For quantitative real time PCR, preparation of recombinant and nuclear protein, EMSA, immunoblot analysis, and ChiP assay, the methods are described in the Supplemental Material.

\section{Acknowledgments}

We thank Shirley Pease (Laboratory Animal Resource, Caltech) for transgenic production, Marcello Coletta for technical help in 
denervation experiments, Leslie Dunipace for technical assistance, Dr. Carlos Lois for parental lentiviral vectors, Dr. Cathy Yuh for help in Affinity Chromatography procedures, Dr. Dario Coletti for help in mice leg electroporation, Hiroke Shizuya for mouse BAC library, Diane Trout for MUSSAGL and genome comparison figures; and Dr. Johannes Graumann for MudPIT analysis performed in the Caltech Beckman Institute PEL center. We are grateful to Drs. C. Berkes and Stephen Tapscott for valuable discussion and exchange of reagents results prior to publication. We also acknowledge Dr. Pier Lorenzo Puri for helpful discussion. This work was supported by NIH, DOE, and NASA grants to B.J.W. and Fondation Leducq, BMW, Duchenne Parent Project, FIRB, and EcMyoAmp grants to G.C.

\section{References}

Berberich, C., Durr, I., Koenen, M., and Witzemann, V. 1993. Two adjacent $\mathrm{E}$ box elements and a M-CAT box are involved in the muscle-specific regulation of the rat acetylcholine receptor beta subunit gene. Eur. J. Biochem. 216: 395-404.

Berkes, C.A., Bergstrom, D.A., Penn, B.H., Seaver, K.J., Knoepfler, P.S., and Tapscott, S.J. 2004. Pbx marks genes for activation by MyoD indicating a role for a homeodomain protein in establishing myogenic potential. Mol. Cell 14: 465-477.

Blagden, C.S., Fromm, L., and Burden, S.J. 2004. Accelerated response of the myogenin gene to denervation in mutant mice lacking phosphorylation of myogenin at threonine 87 . Mol. Cell. Biol. 24: 1983-1989.

Blais, A., Tsikitis, M., Acosta-Alvear, D., Sharan, R., Kluger, Y., and Dynlacht, B.D. 2005. An initial blueprint for myogenic differentiation. Genes \& Dev. 19: 553-569.

Buonanno, A., Apone, L., Morasso, M.I., Beers, R., Brenner, H.R., and Eftimie, R. 1992. The MyoD family of myogenic factors is regulated by electrical activity: Isolation and characterization of a mouse Myf-5 cDNA. Nucleic Acids Res. 20: 539-544.

Buonanno, A., Edmondson, D.G., and Hayes, W.P. 1993. Upstream sequences of the myogenin gene convey responsiveness to skeletal muscle denervation in transgenic mice. Nucleic Acids Res. 21: 5684-5693.

Cheng, T.C., Wallace, M.C., Merlie, J.P., and Olson, E.N. 1993. Separable regulatory elements governing myogenin transcription in mouse embryogenesis. Science 261: 215-218.

Cohen, T.J., Waddell, D.S., Barrientos, T., Lu, Z., Feng, G., Cox, G.A., Bodine, S.C., and Yao, T. 2007. The histone deacetylase HDAC4 connects neural activity to muscle transcriptional reprogramming. J. Biol. Chem. 282: 33752-33759.

Coles, L.S., Diamone, P., Occhiodoro, F., Vadas, M.A., and Shannon, M.F. 2000. An ordered array of cold shock domain repressor elements across tumor necrosis factor responsive elements of the granulocyte-macrophage colony-stimulating factor promoter. J. Biol. Chem. 275: 14482-14493.

Coles, L.S., Lambrusco, L., Burrows, J., Hunter, J., Diamond, P., Bert, A.G., Vadas, M.A., and Goodall, G.J. 2005. Phosphorylation of cold shock domain/Y box proteins by ERK2 and GSK3 $\beta$ and repression of the human VEGF promoter. FEBS Lett. 579: 5372-5378.

Cornelison, D.D. and Wold, B.J. 1997. Single-cell analysis of regulatory gene expression in quiescent and activated mouse skeletal muscle satellite cells. Dev. Biol. 191: 270-283.

Edmondson, D.G., Cheng, T.C., Cserjesi, P., Chakraborty, T., and Olson, E.N. 1992. Analysis of the myogenin promoter reveals an indirect pathway for positive autoregulation mediated by the muscle-specific enhancer factor MEF-2. Mol. Cell. Biol. 12: 3665-3677.
Eftimie, R., Brenner, H.R., and Buonanno, A. 1991. Myogenin and MyoD join a family of skeletal muscle genes regulated by electrical activity. Proc. Natl. Acad. Sci. 88: 1349-1353.

Fontaine, B., Sasson, D., Buckingham, M., and Changeux, J.P. 1988. Detection of nicotinic acetylcholine receptor a-subunit mRNA by in situ hybridization at neuromuscular junctions of 15-day old chick striate muscles. EMBO J. 7: 603609.

Gibney, G. and Buonanno, A. 1995. Analysis of neural-responsive myogenin upstream sequences by myoblast implantation. Dev. Biol. 172: 614-624.

Goldhamer, D.J., Brunk, B.P., Faerman, A., King, A., Shani, M., and Emerson Jr., C.P. 1995. Embryonic activation of the myoD gene is regulated by a highly conserved distal control element. Development 121: 637-649.

Goldman, D. and Staple, J. 1989. Spatial and temporal expression of acetylcholine receptor RNAs in innervated and denervate rat soleus muscle. Neuron 3: 219-228.

Graumann, P.L. and Marahiel, M.A. 1998. Superfamily of proteins that contain the coldshock domain. Trends Biochem. Sci. 23: 286-290.

Graumann, J., Dunipace, L.A., Seol, J.H., McDonald, W.H., Yates III, J.R., Wold, B.J., and Deshaies, R.J. 2004. Applicability of tandem affinity purification MudPIT to pathway proteomics in yeast. Mol. Cell. Proteomics 3: 226-237.

Grounds, M.D., Garrett, K.L., Lai, M.C., Wright, W.E., and Beilharz, M.W. 1992. Identification of skeletal muscle precursor cells in vivo by use of MyoD1 and myogenin probes. Cell Tissue Res. 267: 99-104.

Gundersen, K., Rabben, I., Klocke, B.J., and Merlie, J.P. 1995. Overexpression of Myogenin in muscles of transgenic mice: Interaction with Id-1, negative crossregulation of myogenic factors, and induction of extrasynaptic acetylcoline receptor expression. Mol. Cell. Biol. 15: 7127-7134.

Hannon, K., Smith II, C.K., Bales, K.R., and Santerre, R.F. 1992. Temporal and quantitative analysis of myogenic regulatory and growth factor gene expression in the developing mouse embryo. Dev. Biol. 151: 137-144.

Hasty, P., Bradley, A., Morris, J.H., Edmondson, D.G., Venuti, J.M., Olson, E.N., and Klein, W.H. 1993. Muscle deficiency and neonatal death in mice with a targeted mutation in the myogenin gene. Nature 364: 501-506.

Knapp, J.R., Davie, J.K., Myer, A., Meadows, E., Olson, E.N., and Klein, W.H. 2006. Loss of myogenin in postnatal life leads to normal skeletal muscle but reduced body size. Development 133: 601-610.

Kohno, K., Izumi, H., Uchiumi, T., Ashizuka, M., and Kuwano, M. 2003. The pleiotropic functions of the Y-box-binding protein, YB-1. Bioessays 25: 691-698.

Kostrominova, T.Y., Macpherson, P.C.D., Carlson, B.M., and Goldman, D. 2000. Regulation of myogenin protein expression in denervated muscles from young and old rats. Am. J. Physiol. Regul. Integr. Comp. Physiol. 279: R179-R188.

Lassar, A.B., Buskin, J.N., Lockshon, D., Davis, R.L., Apone, S., Hauschka, S.D., and Weintraub, H. 1989. MyoD is a sequence-specific DNA binding protein requiring a region of myc homology to bind to the muscle creatine kinase enhancer. Cell 58: 823-831.

Lois, C., Hong, E.J., Pease, S., Brown, E.J., and Baltimore, D. 2002. Germline transmission and tissue-specific expression of transgenes delivered by lentiviral vectors. Science 295: 868-872.

Macpherson, P., Kostrominova, T., Tang, H., and Goldman, D. 2002. Protein kinase $C$ and calcium/calmodulin-activated protein kinase II (CaMK II) suppress nicotinic acetylcholine receptor gene expression in mammalian muscle. A specific 
role for CaMK II in activity-dependent gene expression. I. Biol. Chem. 277: 15638-15646.

Macpherson, P.C., Cieslak, D., and Goldman, D. 2006. Myogenin-dependent $\mathrm{nAChR}$ clustering in aneural myotubes. Mol. Cell. Neurosci. 31: 649-660.

Martinenghi, S., Cusella De Angelis, G., Biressi, S., Amadio, S., Bifari, F., Roncarolo, M.G., Bordignon, C., and Falqui, L. 2002. Human insulin production and amelioration of diabetes in mice by electrotransfer-enhanced plasmid DNA gene transfer to the skeletal muscle. Gene Ther. 21: 1429-1437.

Mastrangelo, M. and Kleene, K.C. 2000. Developmental expression of Y-box protein $1 \mathrm{mRNA}$ and alternatively spliced Ybox protein 3 mRNAs is spermatogenic cells in mice. Mol. Hum. Reprod. 6: 779-788.

Matsumoto, K. and Wolffe, A.P. 1998. Gene regulation by Y-box proteins: Coupling control of transcription and translation. Trends Cell Biol. 8: 318-323.

Mejat, A., Ramond, F., Bassel-Duby, R., Khochbin, S., Olson, E.N., and Schaeffer, L. 2005. Histone deacetylase 9 couples neuronal activity to muscle chromatin acetylation and gene expression. Nat. Neurosci. 8: 313-321.

Mendelzon, D., Changeux, J.P., and Nghiem, H.O. 1994. Phosphorylation of myogenin in chick myotubes: Regulation by electrical activity and by protein kinase C. Implications for acetylcholine receptor gene expression. Biochemistry 33: 2568-2575

Mortazavi, A., Thompson, E.C., Garcia, S.T., Myers, R.M., and Wold, J.B. 2006. Comparative genomics modeling of the NRSF/REST repressor network: From single conserved sites to genome-wide repertoire. Genome Res. 16: 1208-1221.

Musarò, A., Cusella De Angelis, M.G., Germani, A., Ciccarelli, C., Molinaro, M., and Zani, B.M. 1995. Enhanced expression of myogenic regulatory genes in aging skeletal muscle. Exp. Cell Res. 221: 241-248.

Nabeshima, Y., Hanaoka, K., Hayasaka, M., Esumi, E., Li, S., Nonaka, I., and Nabeshima, Y. 1993. Myogenin gene disruption results in perinatal lethality because of severe muscle defect. Nature 364: 532-535.

Penes, M.C., Li, X., and Nagy, J.I. 2005. Expression of zonula occludens-1 (ZO-1) and the transcription factor ZO-1-associated nucleic acid-binding protein (ZONA-B)-MSY3 in glial cells and colocalization at oligodendrocyte and astrocyte gap junctions in mouse brain. Eur. J. Neurosci. 22: 404-418.

Piette, J., Bessereau, J.L., Huchet, M., and Changeux, J.P. 1990. Two adjacent MyoD1-binding sites regulate expression of the acetylcholine receptor $\alpha$-subunit gene. Nature 345: 353355.

Prody, C.A. and Merlie, J.P. 1991. A developmental and tissuespecific enhancer in the mouse skeletal muscle acetylcholine receptor $\alpha$-subunit gene regulated by myogenic factors. J. Biol. Chem. 266: 22588-22596.

Sanes, J.R. and Lichtman, J.W. 2001. Induction, assembly, maturation and maintenance of a postsynaptic apparatus. Nat. Rev. Neurosci. 2: 791-805.

Sapru, M.K., Gao, J.P., Walke, W., Burmeister, M., and Goldman, D. 1996. Cloning and characterization of a novel transcriptional repressor of the nicotinic acetylcholine receptor

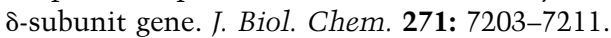

Sassoon, D., Lyons, G., Wright, W.E., Lin, V., Lassar, A., Weintraub, H., and Buckingham, M. 1989. Expression of two myogenic regulatory factors myogenin and MyoD1 during mouse embryogenesis. Nature 341: 303-307.

Shannon, M.F., Coles, L.S., Vadas, M.A., and Cockeril, P.N. 1997. Signals for activation of the GM-CSF promoter and enhancer in T cells. Crit. Rev. Immunol. 17: 301-323.

Sourisseau, T., Georgiadis, A., Tsapara, A., Ali, R.R., Pestell, R.,
Matter, K., and Balda, M.S. 2006. Regulation of PCNA and cyclin D1 expression and epithelial morphogenesis by the ZO-1-regulated transcription factor ZONAB/DbpA. Mol. Cell. Biol. 26: 2387-2398.

Spitz, F., Demignon, J., Porteu, A., Kahn, A., Concordet, J.P., Daegelen, D., and Maire, P. 1998. Expression of myogenin during embryogenesis is controlled by Six/sine oculis homeoproteins through a conserved MEF3 binding site. Proc. Natl. Acad. Sci. 95: 14220-14225.

Tang, H. and Goldman, D. 2006. Activity-dependent gene regulation in skeletal muscle is mediated by a histone deacetylase (HDAC)-Dach-2-myogenin signal transduction cascade. Proc. Nat1. Acad. Sci. 103: 16977-16982.

Venuti, J.M., Morris, J.H., Vivian, J.L., Olson, E.N., and Klein, W.H. 1995. Myogenin is required for late but not early aspects of myogenesis during mouse development. J. Cell Biol. 128: $563-576$.

Walke, W., Xiao, G., and Goldman, D. 1996. A dual function activity-dependent, muscle-specific enhancer from rat nicotinic acetylcholine receptor $\delta$-subunit gene. J. Neurobiol. 31: 359-369.

Wright, W.E., Sasson, D.A., and Lin, V.K. 1989. Myogenin, a factor regulating myogenesis, has a domain homologous to MyoD. Cell 56: 607-617.

Yee, S.P. and Rigby, P.W. 1993. The regulation of myogenin gene expression during the embryonic development of the mouse. Genes \& Dev. 7: 1277-1289. 


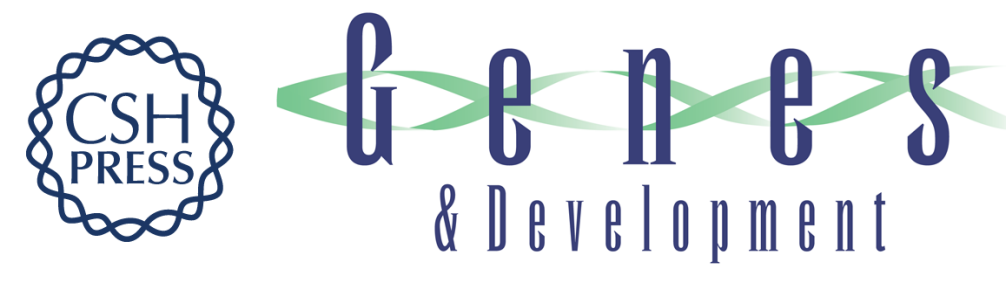

\section{A highly conserved molecular switch binds MSY-3 to regulate myogenin repression in postnatal muscle}

Libera Berghella, Luciana De Angelis, Tristan De Buysscher, et al.

Genes Dev. 2008, 22:

Access the most recent version at doi:10.1101/gad.468508

Supplemental http://genesdev.cshlp.org/content/suppl/2008/08/05/22.15.2125.DC1
Material

References This article cites 52 articles, 21 of which can be accessed free at:

http://genesdev.cshlp.org/content/22/15/2125.full.html\#ref-list-1

License

Email Alerting

Receive free email alerts when new articles cite this article - sign up in the box at the top

Service

right corner of the article or click here.

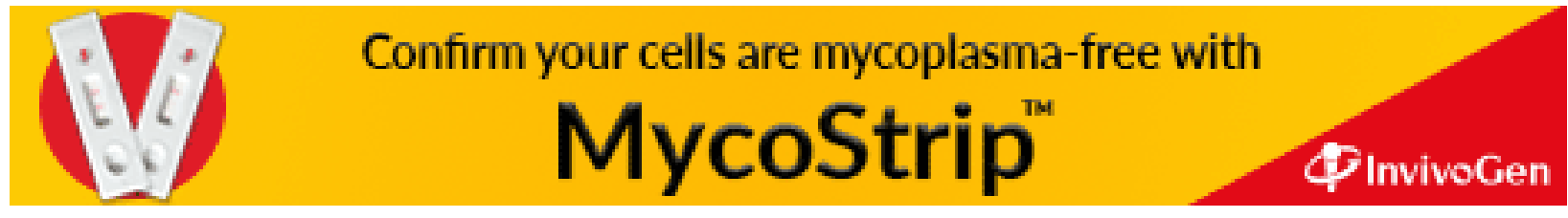

